Proceedings of the International School and Conference on Optics and Optical Materials, ISCOM07, Belgrade, Serbia, September 3-7, 2007

\title{
Stable One-Dimensional Dissipative Solitons in Complex Cubic-Quintic Ginzburg-Landau Equation
}

\author{
N.B. Aleksic ${ }^{a, *}$, G. Pavlovic ${ }^{b}$, B.N. Aleksic ${ }^{c}$ \\ AND V. SKARKA ${ }^{d}$ \\ ${ }^{a}$ Institute of Physics, Pregrevica 118, 11001 Belgrade, Serbia \\ ${ }^{b}$ Faculty of Physics, University of Belgrade, Serbia \\ ${ }^{c}$ Faculty of Electrical Engineering, University of Belgrade, Serbia \\ ${ }^{d}$ Laboratoire POMA, UMR 6136 CNRS, University of Angers
}

2, Boulevard Lavoisier, 49045 Angers, France

The generation and nonlinear dynamics of one-dimensional optical dissipative solitonic pulses are examined. The variational method is extended to complex dissipative systems, in order to obtain steady state solutions of the $(1+1)$-dimensional complex cubic-quintic Ginzburg-Landau equation. A stability criterion is established fixing a domain of dissipative parameters for stable steady state solutions. Following numerical simulations, evolution of any input pulse from this domain leads to stable dissipative temporal solitons. Analytical predictions are confirmed by numerical evolution of input temporal pulses towards stable dissipative solitons.

PACS numbers: 45.65.Sf, 45.65.Tg

\section{Introduction}

The possibility of generating soliton structures in nonlinear media is of considerable interest due to potential applications in all-optical information processing and transport. In telecommunication, temporal solitons are carriers in dispersion compensated optical fiber transmission systems [1,2]. Bright temporal soliton is generated compensating anomalous group dispersion by cubic (Kerr) nonlinearity. Such a few-parameters family of solitons is well described by $(1+1)$-dimensional nonlinear Schrödinger equation; one transverse dimension corresponds to the time $t$ and the propagation coordinate is $z$ [3]. A prerequisite to establish a bridge between the theory and the experiment is to consider real systems that are generally

${ }^{*}$ corresponding author; e-mail: aleksic@phy.bg.ac.yu 
dissipative. In such systems, the solitonic structure can be preserved if appropriate gains match linear and nonlinear losses [4]. In order to stabilize this dissipative soliton the saturating nonlinearity containing also a quintic term of opposite signs is required. As a consequence, the few-parameters family of solutions is reduced to a one fixed solution for a given set of dissipative parameters. Here we investigate one-dimensional complex cubic-quintic Ginzburg-Landau equation (CQGLE) describing various dissipative systems. Except for particular sets of parameters, there are no exact analytical solutions of CQGLE [5]. Such complex systems are treated mainly numerically [4]. However, in order to have a better physical insight into the problem, an analytical approach even though approximate is needed. We extended the variation approach to complex Ginzburg-Landau equation [6]. Based on this variational approach an analytical stability criterion for steady state solutions of Ginzburg-Landau equation is obtained. If such stable solutions are taken as input for numerical simulations, they evolve into solitons. We demonstrate that numerical evolution always leads to stable dissipative one-dimensional solitons. The stability domain of dissipative parameters is established for various types of dissipative temporal solitons.

\section{Formulation of the problem}

The $(1+1)$-dimensional complex CQGLE for the normalized complex field envelope $E$, contains a Laplacian describing the anomalous group velocity dispersion in time $t$

$$
\mathrm{i} \frac{\partial E}{\partial z}+\frac{1}{2} \frac{\partial^{2} E}{\partial t^{2}}+|E|^{2} E+v|E|^{4} E=\mathbb{Q}
$$

Parameter $v$ is negative. Without lost of generality we take $v=-1$. Terms denoted by $\mathbb{Q}$ are all dissipative

$$
\mathbb{Q}=\mathrm{i} \delta E+\mathrm{i} \varepsilon|E|^{2} E+\mathrm{i} \mu|E|^{4} E+\mathrm{i} \beta \frac{\partial^{2} E}{\partial t^{2}} .
$$

The stability of the pulse background involves the linear loss, thus, the parameter $\delta$ must be negative [7]. Parameters $\varepsilon$ and $\mu$ are associated respectively with cubic and quintic gain-loss terms. Last term in Eq. (2) corresponds to spectral filtering. Thus, a simultaneous balance of dispersion with self-focusing and gain with loss is the prerequisite for generation of dissipative temporal solitons.

In order to establish the variational approach for CQGLE we construct the trial function

$$
E=A \exp \left(-\frac{(t-a)^{2}}{2 T^{2}}+\mathrm{i} C(t-a)^{2}+\mathrm{i} V(t-a)+\mathrm{i} \Psi\right) .
$$

Such a trial function is the functional of amplitude $A=A(z)$, temporal pulse widths $T=T(z)$, wave front curvatures, i.e., chirp $C=C(z)$, frequency shift corresponding to "speed" of a pulse "moving in time" $V=V(z)$, coordinate of the field maximum $a=a(z)$ and phase $\Psi=\Psi(z)$. Each of these functions is optimized giving one of six Euler-Lagrange equations averaged over coordinate $t$ : 


$$
\frac{\mathrm{d}}{\mathrm{d} z}\left(\frac{\partial L_{\mathrm{c}}}{\partial \eta}\right)-\frac{\partial L_{\mathrm{c}}}{\partial \eta}=Q_{n} \equiv 2 \Re \int \mathbb{Q} \frac{\partial E^{*}}{\partial \eta} \mathrm{d} t
$$

where the real part is labeled $\Re$. The conservative Lagrangian

$$
\mathbb{L}_{\mathrm{c}}=\frac{\mathrm{i}}{2}\left(E \frac{\partial E^{*}}{\partial z}-E^{*} \frac{\partial E}{\partial z}\right)+\frac{1}{2}\left|\frac{\partial E}{\partial t}\right|^{2}-\frac{|E|^{4}}{2}+\frac{|E|^{6}}{3}
$$

is averaged $L_{\mathrm{c}}=\int \mathbb{L}_{\mathrm{c}} \mathrm{d} t$. This averaged Lagrangian reads

$$
L_{\mathrm{c}}=\left[\Psi^{\prime}+\frac{1}{2}\left(C^{\prime}+2 C^{2}\right) T^{2}-V a^{\prime}+\frac{1}{4 T^{2}}-\frac{A^{2}}{2 \sqrt{2}}+\frac{A^{4}}{3 \sqrt{3}}+\frac{V^{2}}{2}\right] P
$$

where prime indicates a derivative with respect to the coordinate $z$. The beam power $P=\sqrt{\pi} A^{2} T$ is no more conserved in dissipative systems as can be seen from the variation with respect to the phase $[3,6]$. The right hand side of the corresponding Euler-Lagrange Eqs. (4) contains all dissipative terms.

Within variational approximation, to the partial differential CQGLE corresponds a set of six coupled first-order differential equations (FODE):

$$
\begin{aligned}
& \frac{\mathrm{d} A}{\mathrm{~d} z}=\delta A+\frac{5}{4} \frac{\varepsilon}{T_{*}^{2} A_{*}^{2}} A^{3}+\frac{\mu}{T_{*}^{2} A_{*}^{4}} A^{5}-\beta \frac{A}{T^{2}}-C A-\beta V^{2} A \equiv \mathbb{A}, \\
& \frac{\mathrm{d} T}{\mathrm{~d} z}=2 T C-\frac{\varepsilon}{2 T_{*}^{2} A_{*}^{2}} T A^{2}-\frac{\mu}{2 T_{*}^{2} A_{*}^{4}} T A^{4}+\frac{\beta}{T}-4 \beta T^{3} C^{2} \equiv \mathbb{T}, \\
& \frac{\mathrm{d} C}{\mathrm{~d} z}=-2 C^{2}+\frac{1}{2 T^{4}}-\frac{1}{2 T_{*}^{2} A_{*}^{2}} \frac{A^{2}}{T^{2}}+\frac{1}{2 T_{*}^{2} A_{*}^{4}} \frac{A^{4}}{T^{2}}-4 \frac{\beta}{T^{2}} C \equiv \mathbb{C}, \\
& \frac{\mathrm{d} V}{\mathrm{~d} z}=-2 \frac{\beta}{T^{2}}\left(1+4 C^{2} T^{4}\right) V \equiv \mathbb{V}, \\
& \frac{\mathrm{d} a}{\mathrm{~d} z}=V\left(1-2 \beta C T^{2}\right)
\end{aligned}
$$

and

$$
\frac{\mathrm{d} \psi}{\mathrm{d} z}=-\frac{1}{2 T^{2}}+\frac{5}{4 T_{*}^{2} A_{*}^{2}} A^{2}-\frac{1}{T_{*}^{2} A_{*}^{4}} A^{4}+\frac{V^{2}}{2}+2 \beta C\left(1-2 V^{2} T^{2}\right),
$$

where $A_{*}=3^{3 / 4} / 2^{5 / 4}$ and $T_{*}=2 \sqrt{2} / 3^{3 / 4}$.

The steady state solutions are obtained from Eqs. (7)-(10) for right hand side equal to zero $\mathbb{A}=\mathbb{T}=\mathbb{C}=\mathbb{V}=0$. Expanding the steady state with respect to the largest of small dissipative parameters $\theta=\max (|\beta|,|\delta|,|\varepsilon|,|\mu|$, we obtain the amplitude $A=A_{0}+\mathcal{O}\left(\theta^{2}\right)$, temporal width $T=T_{0}+\mathcal{O}\left(\theta^{2}\right)$, and chirp $C=C_{1}+\mathcal{O}\left(\theta^{3}\right)$. As in the conservative systems, the temporal width $T_{0}=T_{*} A_{*}^{2} /\left(A_{0} \sqrt{A_{*}^{2}-A_{0}^{2}}\right)$, power $P_{0}=\left(\sqrt{\pi} T_{*} A_{*}^{2}\right) A_{0} / \sqrt{A_{*}^{2}-A_{0}^{2}}$ and propagation constant $\Omega=\mathrm{d} \psi / \mathrm{d} z=A_{0}^{2}\left(3-2 A_{0}^{2} / A_{*}^{2}\right) /\left(4 A_{*}^{2} T_{*}^{2}\right)+\mathcal{O}\left(\theta^{2}\right)$ depend only on the amplitude $[4,6]$. Variationally obtained families of conservative steady state solutions reduce to a fixed double solution for a given set of dissipative parameters. Indeed, we get the steady state amplitude having two discrete values $A^{+}$and $A^{-}$: 


$$
A_{0}^{ \pm}=A_{*} \sqrt{\frac{(\beta-2 \varepsilon) \pm \sqrt{(\beta-2 \varepsilon)^{2}-4 \delta T_{*}^{2}(3 \mu+2 \beta)}}{3 \mu+2 \beta}}<A_{*} .
$$

If $\varepsilon<\varepsilon_{\mathrm{c}}=\beta / 2-2 \delta T_{*}^{2}$ solution $A_{0}^{+}$exists in the region $\mu>\mu_{2}$ and $\mu>\mu_{1}$ for $\varepsilon>\varepsilon_{\mathrm{c}}$ where $\mu_{1}=(\beta-2 \varepsilon)^{2} / 12 \delta T_{*}^{2}-2 \beta / 3$ and $\mu_{2}=-4\left(\varepsilon+\delta T_{*}^{2}\right) / 3$. The solution $A_{0}^{-}$exists only for $\varepsilon>\varepsilon_{\mathrm{c}}$ and $\mu_{1}<\mu<\mu_{2}$. A double solution $\left(A_{0}^{-}>A_{0}^{+}\right)$exists for a cubic gain $(\varepsilon>0)$ and a quintic loss $(\mu<0)$ in the $(\varepsilon, \mu)$ domain between $\mu_{1}$ and $\mu_{2}$ in Fig. 1. The striking difference with conservative systems is the nonzero wave front curvature $[6,7]$ :

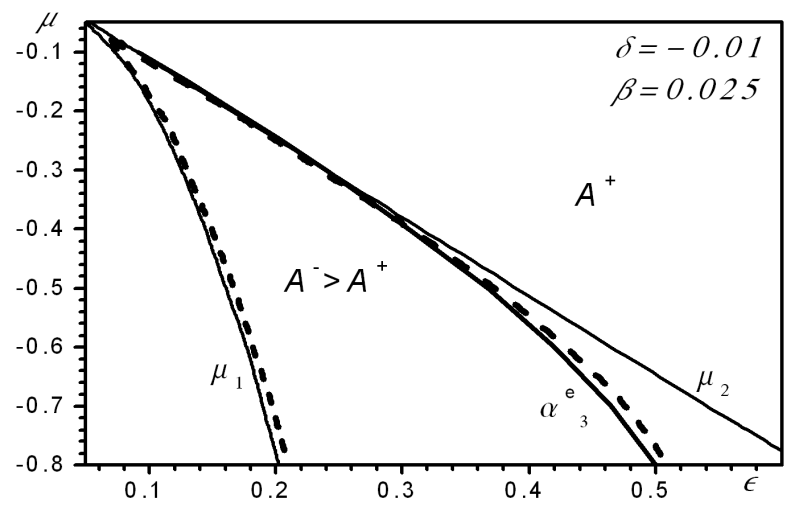

Fig. 1. Analytically (solid lines) and numerically (dashed lines) obtained domain of stability of steady states.

$$
C=\frac{1}{4 T_{*}^{2} A_{*}^{4}} A_{0}^{2}\left[(\varepsilon-2 \beta) A_{*}^{2}+(\mu+2 \beta) A_{0}^{2}\right]
$$

and zero value of "speed" $V=0$, for $\beta \neq 0$.

\section{Stability analysis}

To be a soliton a steady state solution must be stable. In order to check the stability of solutions, we applied our stability criterion based on the variational approach and the method of Lyapunov exponents for Eqs. (7)-(10) [8]. The Jacobi matrix is built from derivatives of terms $\mathbb{A}, \mathbb{T}, \mathbb{C}$, and $\mathbb{V}$ with respect to amplitude $A$, widths $T$, curvatures $C$ and "speed" $V$, taken in steady state. Eigenvalues of Jacobi matrix are determined by equation

$$
\left(\lambda^{3}+\alpha_{1} \lambda^{2}+\alpha_{2} \lambda+\alpha_{3}\right)\left(\lambda+\alpha_{4}\right)=0,
$$

where

$$
\begin{aligned}
& \alpha_{1}=3 A_{0}^{2}\left[(11 \mu-8 \beta) A_{0}^{2}+(8 \beta-2 \varepsilon) A_{*}^{2}\right] /\left(4 T_{*}^{2} A_{*}^{4}\right)+\mathcal{O}\left(\theta^{2}\right), \\
& \alpha_{2}=A_{0}^{4}\left(A_{*}^{2}-A_{0}^{2}\right) /\left(A_{*}^{6} T_{*}^{4}\right)+\mathcal{O}\left(\theta^{2}\right), \quad \alpha_{4}=2 \beta / T_{0}^{2}+\mathcal{O}\left(\delta_{0}^{2}\right)
\end{aligned}
$$

and

$$
\alpha_{3}=A_{0}^{6}\left(A_{*}^{2}-A_{0}^{2}\right)\left[4 A_{*}^{4}(\beta-2 \varepsilon)+2(9 \mu-6 \beta+4 \varepsilon) A_{*}^{2} A_{0}^{2}\right.
$$




$$
\left.+(8 \beta-33 \mu) A_{0}^{4}\right] /\left(2 T_{*}^{6} A_{*}^{12}\right)+\mathcal{O}\left(\theta^{2}\right) .
$$

Following Lyapunov's method, the steady state solutions of four coupled FODE (7)-(10) are stable if and only if the real part of solutions $\lambda$ of equation are negative [8]. This is fulfilled when the Hurwitz conditions are satisfied: $\alpha_{2}>0, \alpha_{3}>0$, $\alpha_{123}=\alpha_{1} \alpha_{2}-\alpha_{3}>0$ and $\alpha_{4}>0$. The last condition is crucial and it is realized when $\beta>0$. The condition $\alpha_{2}>0$ is always satisfied for stationary solutions $A_{0}^{ \pm}<A_{*}$. However, only the solutions $A^{-}$satisfy $\alpha_{3}>0$ and $\alpha_{123}>0$ for $\theta<1$. As a consequence, in the $(\varepsilon, \mu)$ domain in Fig. 1 only $A^{-}$solution is stable. The stability criterion for steady state solutions of CQGLE is explicitly expressed as

$$
\begin{aligned}
& \beta>0, \\
& \varepsilon>\beta / 2-2 \delta T_{*}^{2}
\end{aligned}
$$

and

$$
(\beta-2 \varepsilon)^{2} / 12 \delta T_{*}^{2}-2 \beta / 3<\mu<-4\left(\varepsilon+\delta T_{*}^{2}\right) / 3
$$

Solving exactly but parametrically Eqs. (7)-(10) and using Hurwitz's conditions the exact curve for $\alpha_{3}^{e}$ is drawn in Fig. 1 limiting the domain of dissipative parameters for stable stationary states. In order to check the validity of analytical prediction of the domain of stable steady states solutions based on the stability criterion Eqs. (7)-(10), numerical simulations of Eq. (1) was performed taking as input dissipative parameters from this domain. Although such initial states are not yet solitons, the system will after some evolution self-organize into stable dissipative temporal soliton.

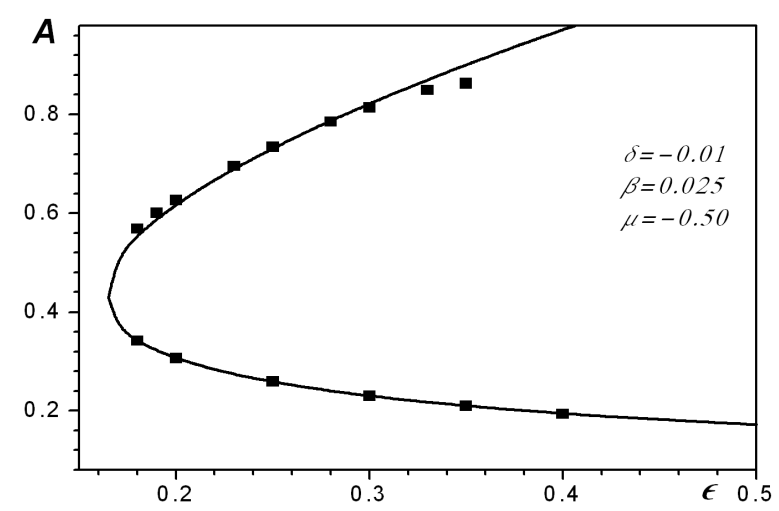

Fig. 2. Numerical (squares) and analytical (solid line) bifurcation curve of amplitude $A$ versus parameter $\varepsilon$.

Starting from Gaussian pulse instead of stable steady state is equivalent to perturbation of the soliton with respect to the amplitude, width, curvature, and frequency shift. Taking into account that the dissipative soliton is exceptionally robust, such perturbations will be damped, bringing the system back to solitonic solution which corresponds to the dynamical equilibrium. Such an evolution cor- 
responds to the transition from the stable branch of the variational bifurcation curve to the numerical bifurcation curve in Fig. 2.

In order to test the robustness by numerical simulations of Eq. (1), an input Gauss pulse is chosen from the stable region of material parameters, with finite initial "speed", and no initial curvature. The initial frequency shift will produce a pulse drift following time axis (see Fig. 3a).

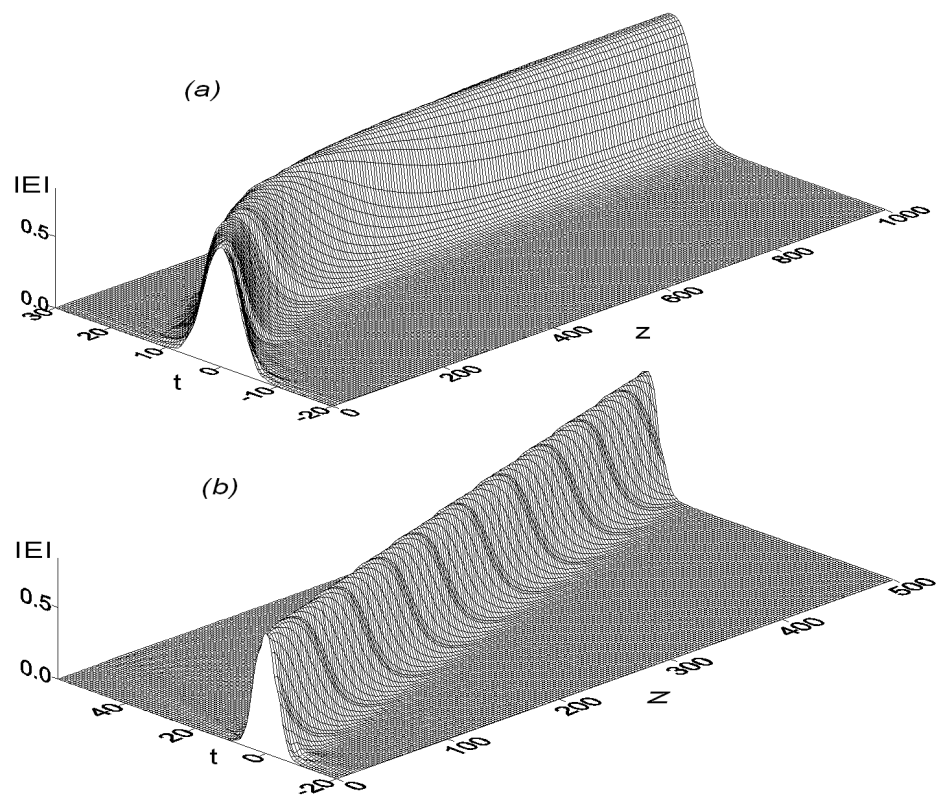

Fig. 3. Evolution of the Gauss pulse for: $\delta=-0.01, \varepsilon=0.2, \mu=-0.3, C=0, V=0.1$; $\beta=0.02$ (a) and $\beta=0$ (b).

However, such a shift is damped to zero since the steady state has always $V=0$ as can be seen from Eq. (10). If now the parameter $\beta$ is zero keeping all other parameters the same, the simulations show an undamped drift (see Fig. 3b). Indeed, for $\beta=0, \alpha_{4}$ cannot be positive. Therefore, Hurwitz's conditions are not satisfied. As a consequence, in order to have the stable stationary state, the parameter $\beta$ must be larger than zero.

\section{Conclusion}

The stability of soliton solutions of $(1+1)$-dimensional cubic-quintic Ginzburg-Landau equations was studied using variational approach. A stability criterion was established using Hurwitz's conditions not only for the amplitude, width, and curvature, but also for the frequency shift although it is not present in steady states. Based on such a criterion, a stability domain of dissipative parameters is established. Any pulse from this domain taken as the input for the 
numerical simulations will during propagation self-organize into stable dissipative temporal soliton playing the role of an attractor. It is demonstrated that the coefficient $\beta$ of the spectral filtering dissipative term in Eq. (1) must be positive in order to have the stability.

\section{Acknowledgments}

This research was in part supported by French-Serbian cooperation, CNRS/MSCI agreement No. 20504.

\section{References}

[1] I. Gabitov, S.K. Turitsyn, Opt. Lett. 21, 327 (1996).

[2] M. Nakazawa, H. Kubota, Jpn J. Appl. Phys. 34, L681 (1995).

[3] N.N. Akhmediev, A.A. Ankiewicz, Solitons, Nonlinear Pulses and Beams, Chapman and Hall, London 1997.

[4] V. Skarka, V.I. Berezhiani, R. Miklaszewski, Phys. Rev. E 56, 1080 (1997); V. Skarka, V.I. Berezhiani, R. Miklaszewski, Phys. Rev. E 59, 1270 (1999).

[5] N.N. Akhmediev, V.V. Afanasjev, J.M. Soto-Crespo, Phys. Rev. E 53, 1190 (1996).

[6] V. Skarka, N.B. Aleksic, Phys. Rev. Lett. 96, 013903 (2006).

[7] N.B. Aleksic, V. Skarka, D.V. Timotijevic, D. Gauthier, Phys. Rev. A 75, 061802(R) (2007).

[8] G. Nicolis, I. Prigogine, Self-Organization in Nonequilibrium Systems, Wiley, New York 1977. 